Available online on 18.08.2019 at http://jddtonline.info
(c) 2011-18, publisher and licensee JDDT, This is an Open Access article which permits unrestricted
non-commercial use, provided the original work is properly cited

Open $\odot$ Access

Review Article

\title{
Summary and application analysis of clinical herniorrhaphy mesh materials
}

\author{
Yan Liu' ${ }^{1}$, Yanbo Zuo ${ }^{1}$,Zhengwei Jiao ${ }^{2}$, Xiaoshi Jin ${ }^{2 *}$ \\ 1. Medical College of Hebei University, Baoding, Hebei, China, 071000 \\ 2. Affiliated Hospital of Hebei University, Baoding, Hebei, China, 071000
}

\begin{abstract}
It is widely believed in the medical community that the pathological and anatomical basis for the occurrence of external abdominal hernia is the fascia defect, and how to correctly deal with this kind of fascia defect is the key point in the treatment of external abdominal hernia. Theodore Billorth famously said that if we could artificially create dense and tough tissues like fascia and tendons, the secret to the complete healing of hernia would be solved. These similar theories largely guide the research and practice in the treatment of external abdominal hernia. along with the breakthrough progress in the mesh treatment of external abdominal hernia, According to the chemical composition and biological characteristics, the patch materials can be divided into non-absorbable materials, absorbable materials, composite repair materials and biological materials. Methods In this paper, Pubmed database and Chinese journal full-text database literatures were searched,and all mesh materials used in inguinal hernia repair were comprehensively studied, summarized and analyzed in clinical application.
\end{abstract}

Keywords: external abdominal hernia; patch material; conclusion; application

Article Info: Received 11 June 2019; $\quad$ Review Completed 28 July 2019; $\quad$ Accepted 31 July 2019; $\quad$ Available online 18 August
2019

*Address for Correspondence:

Xiaoshi Jin, Affiliated Hospital of Hebei University, Baoding, Hebei, China, 071000

\section{Introduction}

With the continuous progress of modern medical technology, the treatment of hernia diseases has become increasingly standardized and professional, thus opening up a new branch of general surgery--the development of hernia and abdominal wall surgery. Hernia patch repair dates back to 1894 when silver ornaments were used to repair the posterior wall of the inguinal canal. The first application of artificial material metal mesh repair of inguinal hernia was completed by Mr. La ISSN: 2250-1177
Roque in 1919.In 1954,the first polyester mesh was synthesized. In 1962, American Francis Usher used Marlex mesh for inguinal hernia repair, which initiated the first repair of inguinal hernia mesh. However, the real artificial material repair of inguinal hernia started in 1989 when American doctor Lichtenstein[1] proposed the concept of tension-free hernia repair, and later synthetic mesh represented by polypropylene has been widely used in the field of hernia surgery around the world. It has become the 


\section{Liu et al}

gold standard in the treatment of inguinal hernia in many countries. Although artificial mesh has obvious advantages, a series of potential complications caused by inflammatory reactions such as foreign body reactions should not be underestimated. In the process of continuous clinical development and improvement, since the first biological mesh was approved for soft tissue reconstruction in 1999, it has received great research and attention from researchers due to its better histocompatibility and the goal of tissue repair. This article reviews the types, characteristics and application of herniorrhaphy materials.

\section{Non-absorbent material}

Clinical account for more, and can be divided into metallic materials and non-metallic materials.

\subsection{Metal materials}

\subsubsection{Features of metal material mesh}

Silver ornaments were first used to repair the posterior wall of the inguinal canal. In addition, the mesh formed by gold and silver wire ornaments, tantalum yarn and stainless steel rings were also used as hernia repair materials in clinical practice.

\subsubsection{Clinical application effect of metal material mesh}

Tough and durable. However, softness is poor, most patients are uncomfortable, have local fluid retention, have the potential risk of infection and sinus tract after infection.

\subsubsection{Clinical experience and application}

In 1907, a metallic double mesh was used by McGavin, one deep graft under the aponeurotic arch of the transverse abdominis and the other under the aponeurosis of the upper spermatic line of the external oblique. Ball counted 500 cases in 1958, only two of which relapsed,and has rarely been used in clinical practice.

\section{2 non-metallic materials}

Including, nylon, olyethylene, polyester,polypropylene, polytetrafluoroethylene, carbon fiber and so on. At present, nylon(because of its rapid loss of strength in the body) and polyvinyl alcohol sponge(with poor histocompatibility)are no longer used clinically[2].At present, polypropylene mesh, polyester mesh and expanded PTFE mesh are widely studied and applied. Studies on carbon fiber mesh have proved that it has good histocompatibility, good collagen tissue scaffold effect, and can improve the strength of induced connective
Journal of Drug Delivery \& Therapeutics. 2019; 9(4-s):714-720

tissue, so that its clinical application is valued[3], but its long-term application results still need to be observed in clinical and laboratory.

\subsubsection{Polyester patch (PE)}

\subsubsection{Characteristics of PE}

Clinical application began in the 1960s.The main application is polyester mesh (synthetic material mesh), which is low in price. This kind of mesh is soft and elastic with good compliance, but its tensile strength is only $1 / 3$ of that of polypropylene mesh. There was moderate foreign body reaction and strong fibrous tissue hyperplasia.

\subsubsection{Clinical category of PE}

There are mainly,(1)Mersilene mesh, multi-strand mesh,(2)Dacron mesh, single-strand mesh[4].

\subsubsection{Clinical effect of PE}

Polyester mesh is a fiber structure, so its anti-infection ability is general. It is more likely to cause intestinal adhesion and obstruction or even intestinal fistula when placed alone in the abdominal cavity.

\subsubsection{Polypropylene patch (PP)}

\subsubsection{Features of $P P$}

It is woven by polypropylene single fiber or multi-strand fiber, and it is a single-layer mesh structure, which is the preferred repair material for abdominal wall defect at present.

\subsubsection{Clinical category of PP}

Common polypropylene mesh:(1)Marlex mesh, Bard products, single mesh.(2)Prolene network, Ethicon company products, double mesh.(3)Surgipro mesh, American surgical company products, multi-strand mesh. Such mesh is a woven mesh with large mesh holes, which is conducive to the growth of fibrous tissue and can improve the strength and tensile strength of the tissue.White blood cells and macrophages can freely enter the mesh, which is not easy to harbor bacteria, and has good anti-infection ability, so it is not necessary to remove the mesh after infection[5]. However, due to its rough surface and hard texture, it is easy to cause serious complications such as intestinal adhesion or even intestinal fistula when placed in the abdominal cavity, so it cannot be placed in the abdominal cavity alone[6].According to the weight of polypropylene mesh, it can be divided into: weight polypropylene mesh(including more than $70 \%$ 
polypropylene), such as Marlex mesh and Prolene mesh; Light weight polypropylene mesh(including $40 \%$ to $50 \%$ polypropylene),such as Proceed mesh; Super lightweight polypropylene patch(with polypropylene $17 \%$ to $30 \%$ ),as Mpathy patch. Light materials are characterized by good flexibility, small inflammatory response, and are not easy to cause chronic pain[7].

\subsubsection{Clinical application effect of PP}

Compared with other non-absorbable materials, in addition to the above advantages are: the price is relatively low, repair and material related complications are less, the main infection, serous swelling, intestinal adhesion, intestinal leakage and material displacement, material shrinkage. Polypropylene is an ideal hernia repair material with good histocompatibility (with certain anti-infection ability).Its repair is simple, less damage, quick recovery and less complications ${ }^{[8] . D o m e s t i c ~ J i a n-x i o n g ~ T a n g[9] ~ a n d ~ o t h e r s ~}$ report of 256 cases of hernia ring filling type without tension hernia repair were followed up for 1 year: After surgery, the patient recovered from bed at 3 to 6 hours, low thermal $51.6 \%$ within two days postoperatively, postoperative complications, $11.5 \%$ (no infection), $2.7 \%$ of recurrence, due to the reports of operation source comes from the level and time of no more than 10 surgeon operation, so the actual effect of single operation or slightly experienced performer than the statistics will be more low, so has more realistic significance. Hua lei[10] et al reported 102 cases of polypropylene mesh plug and mesh herniorrhaphy with an average operative time of $31.5 \mathrm{~min}, 4-6$ hours after surgery, and 2-3 days of wound pain. Postoperative dysuria occurred in 1 case, wound effusion in 2 cases, no incision infection in 1 case, and foreign body sensation in 9 cases. A total of 89 cases were followed up and 1 case relapsed. In addition, according to Rutkow[11],2403 cases of herniorrhaphy were reported. After postoperative follow-up,11 cases had recurrence, and the recurrence rate was less than $0.5 \%$.

\subsubsection{4 clinical experience and application of PP}

If the hernia ring is filled with a cone-shaped filling(for special cases: because the hernia ring is too small, cut off 1-4 supporting petals in the filling; The hernia ring was too large, The hernia ring was inserted by suturing the two fillers together. The composite hernia was placed with one filling in each of the different hernia rings, and the molded mesh(a non-absorbent material woven by polypropylene monofilament, with good anti-infection and ISSN: 2250-1177 [716] histocompatibility, which could be quickly adhered and fixed with human tissues)was placed behind the spermatic cord to strengthen the posterior wall of the inguinal canal, which was not easy to relapse after surgery. This technique is more suitable for the treatment of human physiological anatomy. Therefore, for patients with chronic cough, chronic constipation and dysuria of prostatic hyperplasia, surgical indications can be relaxed. Due to the simple operation, light injury and short time, this operation is also suitable for elderly patients with cardiovascular and cerebrovascular diseases. It is also applicable to patients with giant hernia ring $(>4 \mathrm{~cm})$, cirrhosis with mild ascites and recurrence after tension-free patch. Skilled identification and grasp of the anatomical structure, fully free mesh to expand the space involved, so that the patch is spread out, the correct placement is the key to the surgery. In order to avoid wound effusion, the thorough hemostasis during the operation should be emphasized first. Secondly, after the placement of the filling materials, the accumulated liquid in the filling materials is absorbed and cleaned. Thirdly, immediately after the surgery, press the wound area with the palm for $5 \mathrm{~min}$,which is better than pressing the wound with the pocket after returning to the ward for $6 \mathrm{~h}$.Fourthly,obese patients are more prone to fat liquefaction, and skin slices can be placed in the wound for $24 \mathrm{~h}$ after surgery for drainage. In addition, the hole in the center of the mesh through the spermatic cord should not be too small, otherwise it will affect the blood circulation of the testis and cause ischemic orchitis or even testicular necrosis ${ }^{[9,10,12,13]}$.

\subsection{3 expanded polytetrafluorethylene(e-PTFE)}

\subsubsection{Characteristics of e-PTFE}

As an inert microporous material, the surface is composed of multi-direction fibers of columnar nodules, so the forces in all directions of the mesh are in balance. In addition to good tensile strength and anti-infection ability, it also has a unique porous microstructure, making it softer, more flexible and more compatible.

\subsubsection{Clinical category of e-PTFE}

The main representative products are Mycro Mesh mesh and Dualmesh mesh produced by Gore company. The first report was reported on 30 cases of hernia repair completed by Wool[14] et al. The MycroMesh mesh is stretched by a special process, with an average diameter of $22 \mu \mathrm{m}$ and an average porosity of $80 \%$,allowing tissue cells to be embedded. It is 


\section{Liu et al}

chemically stable, hydrophobic, flexible, smooth, compliant and extremely durable. Dualmesh mesh surface aperture $<3 \mu \mathrm{m}$,smooth surface, can prevent the growth of cells, and the internal surface of the adhesion does not occur[5].Different forms of e-PTFE patches can be made according to clinical requirements: (1)standard holes $20-25 \mu \mathrm{m}$, with large holes added regularly in the middle.(2)one side is $3 \mu \mathrm{m}$ holes, the other side is a standard hole, both to prevent adhesion and facilitate tissue growth[2].A single-layer, double-sided e-PTFE patch developed by Gore company has a Dualmesh structure on one side, which prevents tissue growth and contacts with internal organs of the abdomen, and MycroMesh on the other side, which allows tissue growth and contact with abdominal wall tissue, thus making the structure more reasonable[6].

\subsubsection{Clinical application effect of e-PTFE}

Li wei[14-15] et al reported that the average operative time of PTFE mesh (275 cases) was 50.5 min.3-6 hours after surgery, the patients recovered their independent movement out of bed, with a recurrence rate of $4.72 \%$.There were 37 cases of recurrent hernia, 5 cases of recurrent hernia after re-repair, and 1 case of postoperative incision infection. Finally, the mesh was removed and cured. Incisional effusion was observed in 4 cases, scrotal effusion in 1 case, traction pain in 7 cases, foreign body sensation in 1 case, and swelling sensation in 5 cases. The anti-infection ability of PTFE mesh is poor compared with polypropylene mesh due to small mesh size $(<10 \mu \mathrm{m})^{[16]}$.

\section{Absorbable materials}

\section{1 characteristics of absorbable materials}

Absorbable hernia repair materials can be gradually dissolved and absorbed in the body over time. This process depends on the nature of the absorbable materials, and the total absorption time is about 3 months.

\section{2 clinical categories of absorbable materials}

At present in clinical use of the main polyglycolic acid and polylactic acid hydroxyacetic acid two kinds of absorbable mesh, its commercial names are Dexon and Vicyle.

\section{3 clinical application effects of absorbable materials}

Absorbable mesh cannot stimulate the body to produce enough fibrous tissue hyperplasia, so it is easy to cause hernia again in the repair site after absorption. Therefore, these two materials are not mature enough for the repair of ISSN: 2250-1177
Journal of Drug Delivery \& Therapeutics. 2019; 9(4-s):714-720

abdominal wall defects. However, when the non-absorbable materials cannot be used for repair due to severe infection, fistula and other conditions, the anti-infection ability of absorbable materials can temporarily restore the continuity of the abdominal wall, which can help patients get through the dangerous period of the disease, and permanent materials can be used for deterministic repair during the second-phase repair. Therefore, repair of rupture and injury of internal organs (such as spleen and kidney)is more commonly used in clinical practice[3].

\section{Composite materials}

\section{1 characteristics of composite materials}

In order to make full use of the characteristics of different materials from the composite material.

\section{2 clinical category and application effect of composite materials}

(1)The polypropylene mesh combined with e-PTFE material patch:the patch has Composix patch(Bard company products),IntrameshT1 patch(French Cousin biotech companies), Motifmesh patch[15](Irish Medchannel company)etc both the advantages of the two materials, such as strengthening anti-adhesion effect, good rigidity and resistance to tension, at the same time because of the stain resistance of e-PTFE patches no strengthened, once the infection is often need to remove the patch[3].Composite material mesh is a typical BardMesh Composix Kugel double-sided mesh produced by Bard company, which is composed of two layers of PP mesh and one layer of e-PTFE. Monofilament PP surface stimulates tissue to grow into the mesh, reducing the risk of recurrence. e-PTFE surface minimizes the adhesion between the tissue and the mesh to protect the vital organs in the abdominal cavity.But its disadvantage is the material is thicker,fixed after the abdominal wall compliance is poor.(2) The combination of PP mesh and absorbable material mesh: this material is PP mesh as the skeleton, and then use the absorbable material composite,its purpose is to reduce the amount of foreign body(PP),anti-adhesion and effective anti-infection.Such materials mainly represented by Proceed net, the latest research and development products, Johnson\&johnson companies in the United States is a lightweight mesh, is divided into four layers, the concrete structure is to embed the cannot absorb the PP mesh together of two layer can absorb oxygen between cyclohexanone, below to add a layer 
of absorbable oxidized regenerated cellulose membrane, polymer membrane for oxygen cyclohexanone in PP mesh and maintain the bond between oxidized regenerated cellulose, the film began cracking within $14 \mathrm{~d}$ after implantation, six months or so completely absorbed. Oxidative regenerated cellulose was absorbed within 14 days after implantation[5].The separated surgical mesh of the multi-layer tissue can effectively avoid the direct contact between the abdominal internal organs and the PP mesh, so as to reduce the adhesion with the internal organs and safely attach to the inner layer of the abdominal wall. At the same time, the mesh is large, light monofilament configuration, free of tetrafluoroethylene, can reduce the adhesion of bacteria, and has good flexibility, easy to integrate with the tissue.

\section{Biological materials (acellular extracellular matrix, AEM)}

\section{1 characteristics of biological materials}

The basic material is the extracellular matrix obtained from allogeneic or Heterogeneous variants tissues after treatment. The technology preparation and use of the idea is raw material using cell technology, get rid of the causes of host immune rejection of all ingredients, completely retained the extracellular matrix and three-dimensional scaffold structure, attract a host cell growth on the scaffolds, secrete new extracellular matrix components, form their own organization, which is by endogenous tissue(intrisic tissue regeneration)to complete the process of defect repair and reconstruction of the organization. It is completely different from the tissue repair process of polymer materials. With the use of non-metallic materials of the accumulation of a large number of cases, for the slurry is swollen, infection, chronic pain, the patch shrinkage, intestinal adhesion, intestinal obstruction, intestinal fistula, and pay more and more attention to problems such as recurrence, this is the current hernia materials scientists constantly explore the improvement and development of composite material, with collagen as the main body of the biological materials for further research of the background to the ${ }^{[17]}$.According to different tissue sources, it can be divided into autologous biological materials, heterogeneous tissue patches and variants tissue patches.

\section{2 types and clinical characteristics of biological materials}

Autologous biomaterials: using autologous tissue slices, there is no antigenicity problem, but due to the large trauma, the graft is easy to degenerate and deform, and there are many complications, It is less used now. Allogeneic tissue patch: the allogeneic dermal tissue is removed from the antigen components, and the three-dimensional structure of the extracellular matrix is retained. The growth and proliferation of the own cells on the allogeneic dermal tissue and the secretion of new extracellular matrix by the new cells are interacted with each other and become part of the own tissue[18].At present there are Alloderm and Permacol of commercialization two patches, Lifecell products in the United States, came from the organization for the body, to cell matrix material, no structural protein, retained the dermal extracellular matrix components, with resistance to infection,prevent adhesion, can increase the intensity of the abdominal wall, the characteristics of more for acute abdominal wall defect and or dirty wound infection abdominal wall defect repair; XenMatrixTM patch by Brennen in the United States and SurgiMendTM graft by Cook Surgical in the United States are two kinds of XenMatrixTM graft and SurgiMendTM graft.The former is derived from pig collagen tissue and the latter is derived from fetal cow skin, with good histocompatibility, and can provide a scaffold for the growth of autologous tissue, which is mostly used for the repair of acute abdominal wall defect.

\section{3 clinical study on biological materials}

In 2014,Bochicchio et al conducted a prospective, randomized and double-blind study.100 patients with inguinal hernia were randomly divided into two groups, and Lichtenstein repair was performed with biological mesh(IHM,Cook Medical)and polypropylene(PP) respectively, The mean follow-up was 12 months. The postoperative chronic pain in IHM group was 4\%,and that in PP group was $6 \%$,which was statistically significant. Agresta and Bdin published study biological patch (Surgisis patch) for laparoscopic repair by peritoneal (TAPP) evaluation, a total of 20 cases of inguinal hernia, intraoperative using fibrin glue fixed patch, postoperative follow-up(14 \pm 5$)$ months on average, no complications such as infection, intestinal adhesion, chronic pain, have appeared in 1 case recurred, analysis can be caused to intraoperative operating problems. The results show that the biological mesh is safe and effective. The Chinese scholar Shen Yingmo et al used acellular tissue matrix material biological mesh to perform tension-free repair for $6 \sim 18$ years old patients with inguinal hernia.30 patients in the experimental group received Lichtenstein 


\section{Liu et al}

plain patch repair with biological mesh.In the control group,30 cases were treated with high ligation of hernia sac.Patients in the two groups were followed up for 14 to 20 months, and there were no complications of incision infection, chronic pain or local foreign body sensation. Postoperative scrotal effusion occurred in 3 patients in the experimental group without recurrence. There were 2 cases of scrotal effusion and 2 cases of recurrence in the control group. The final conclusion is that in the treatment of children and adolescents with inguinal hernia between 6 and 18 years old, the application of biological mesh in plain film tension-free hernia repair has a good effect and does not increase postoperative complications ${ }^{[19]}$.

\section{4 clinical application effects of biological materials}

The advantages of biomaterial mesh and high molecular mesh in tissue repair are: no excessive scar tissue; Chronic inflammation that occurs when no foreign body polymer remains in the body. It has been reported[17] that acellular matrix materials can completely meet the requirements of abdominal wall defect repair in the absence of rejection and tension. Due to the rapid vascularization of the recipient host tissue to the implanted mesh and the resistance to infection, the neoperitoneum can resist the formation of adhesion, and if the implanted mesh is infected or exposed to the wound, local or systemic treatment can be carried out and other key characteristics are of clinical concern.

\subsection{Clinical applications of biological materials}

Combined with the advantages of biomaterial mesh, it can be used in complex and difficult fields.Acute abdominal wall opening such as trauma, acute septal syndrome; Acute abdominal wall infection such as trauma, postoperative infection; Complex abdominal hernias, such as the disappearance of the physiological dome in the abdominal wall, form a second abdominal cavity, mesh infection and intestinal fistula after mesh repair; Dorsal division of fascia; Parastomal hernia. Hiatal hernia, filling therapy for anal fistula, even inguinal hernia in young people, inguinal hernia in children[18] (good efficacy, low recurrence rate, no adverse reactions) can be used.

\section{Conclusions}

When choosing herniorrhaphy, the most skilled and profound operation should be the best choice. It can minimize the occurrence of postoperative complications, especially the recurrence rate and postoperative discomfort.
Journal of Drug Delivery \& Therapeutics. 2019; 9(4-s):714-720

Polypropylene mesh has the advantages of low material price, simple operation and short operation time. Another polypropylene patch and e-PTFE patch, composite materials, absorbable materials and biomaterials patch must be combined with its own characteristics analysis individualized treatment, to take off the cell biological ecms patch as a new kind of absorbable materials in developed countries has been widely used in clinical, the domestic scholars in hernia repair also has a certain clinical experience, its irreplaceable advantages in clinical at the same time, pay more attention to research also suggests that Theodore Billorth words more and more near the feasibility of clinical implementation-completely cured. Due to the characteristics of histocompatibility, almost no infectivity, enhanced abdominal wall strength, almost no chronic pain after surgery, almost no local scleroma or discomfort, almost no recurrence and so on, along with the drop in mass production price, it will definitely push the hernia and abdominal wall surgery to a new height.

\section{References}

1. Lichtenstein IL,Shulman AG.Ambulatory outpatient hernia surgery.Including a new Concept, introducing tension-free repair[J] Int Surg,1986,71(1):1-4.

2 DeBord JR. The historical development of prosthetics in hernia surgery[J].Surg Clin North Am,1998,78(6):973-1006.

3 Yundong Zou.Biocompatibility of mesh materials for inguinal hernia repair[J].Tissue engineering research and clinical rehabilitation in China,2008,12(19): 3705-3708.

4 Gang Wang,Yu Zhao.Progress in clinical application of hernia repair materials[N].Journal of chongqing medical university, 2007,32(1):110-112.

5 Shuang Chen,Bin Yang.A new material for hernia repair[J].Chinese journal of minimally invasive surgery, 2007,7(12):1123,1124.

6 LeBlanc KA.A new method to insert the DualMesh prosthesis for laparoscopicventral herniorrhaphy[J].JSLS, 2002,6(4):349-352.

7 Jiye Li.Types of hernia repair materials and related complications[J]. Journal of clinical surgery, 2006, 14(11):687-689.

8 Yinghua Mi,Ying Liu,Xiang Wang.Application of polypropylene repair material in the treatment of inguinal hernia[J].Tissue engineering research and clinical rehabilitation in China, 2010,30(16):432-434. 


\section{Liu et al}

9 Jianxiong Tang,Ge Chen,Lei Huang.Clinical experience in treating 256 cases of inguinal hernia with hernia ring filling tension-free hernia repair[]].Chinese journal of practical surgery, 2001, 21(2):78-79.

10 Lei Hua,Feng Han.Clinical application of polypropylene mesh plug and mesh in inguinal hernia repair[J].Chinese journal of practical surgery,2001,21(2):84-85.

11 Rutkow IM,Robbins AW,Mesh plug hemia repair:a follow-up report[J].Surgery,1995,117(5):597.

12 Songzhang Ma,Yanqing Li.Hernia ring filling tension-free repair.Journal of clinical surgery[J],1998,6(4):230.

13 Yanchang Chen, Heguang Huang, Fengchun Lu.Clinical application of monofilament polypropylene textile mesh in inguinal hernia repair[J] . Chinese journal of practical surgery.2007,27(7):553-554.

14 Wool NL,StraussAK,Roseman DL.Clinical experimance with the

\section{Journal of Drug Delivery \& Therapeutics. 2019; 9(4-s):714-720}

Gore-Tex soft tissue patch in hernia repair:A preliminary report[J].Preo Inst Med Chicago,1985,38(33):459-462.

15 Wei Li,Yangshen Jiang,Jing Zhang.Comparison of efficacy of three kinds of mesh in tension-free hernia repair[J].Chinese journal of misdiagnosis.2007,17(5):97-98.

16 Wei Li,Yangsheng Jiang,Jingjing Zhang.Comparative study of acellular allogeneic tissue mesh in tension-free hernia repair[]].Chinese journal of medical education,2007, 30(70):49, 50,53 .

17 Songzhang Ma,Progress and clinical application of biological mesh[J].International journal of surgery,2008,35(12):793-795.

18 Haijing Gao,Application of biological mesh in inguinal hernia in children[J].Medical theory and practice,2012,25(2):172-173.

19 Weibing Li,Bo Huang,Progress in the application of biological mesh in repair of external abdominal hernia[J].Journal of practical medical techniques,2016,23(2):165-167. 